\section{Early and late protection from TB}

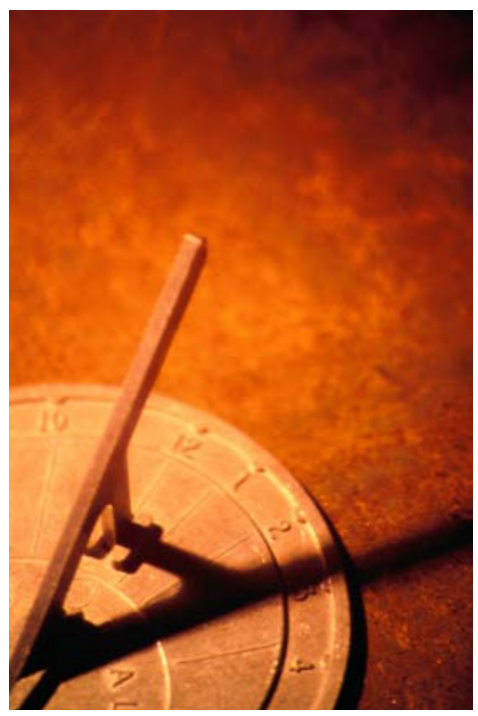

The BCG vaccine (which is the only approved vaccine against tuberculosis (TB)) and other TB vaccines that are currently in clinical trials are designed to protect against infection by incorporating antigens that are expressed early in the disease process. However, they do not prevent the establishment of latent persistent infection or reactivation of clinical disease, which is a major unmet need for infected patients and for reducing further transmission.

Reporting in Nature Medicine, Aagaard and colleagues show that a vaccine comprising antigens that are expressed in the early and late stages of TB combated late-stage infection in both pre- and post-exposure mouse models.

The vaccine (termed H56) comprises a fusion protein (Ag85B-ESAT6 (a 6 kDa early secretory antigenic target)-Rv2660c) and a cationic adjuvant (CAF01). Ag85B and ESAT6 are well-known Mycobacterium tuberculosis antigens that are secreted early in infection; they have previously been shown to provide protective immunity, and an Ag58B-ESAT6 fusion protein is currently in clinical trials.

Rv2660c, which is known to be involved in the stress response, was identified in this study as one of the factors that is expressed at constant levels throughout the stages of infection. The authors proposed that simultaneous vaccination with these three antigens could produce multistage effects, by enabling the immune system to mount a response to both the early and late phases of TB.
In the early stage of TB infection ( $<4$ weeks after exposure), the bacterial load in the lungs of H56-vaccinated mice was similar to that of mice vaccinated with the BCG vaccine. However, in the later stage of infection (24 weeks after exposure), H56-vaccinated mice had significantly lower numbers of bacilli compared to BCGvaccinated mice. Moreover, H56 was an effective booster to BCG, with H56-vaccinated mice having significantly lower bacterial loads 24 weeks after infection compared to mice vaccinated with BCG alone.

Importantly, in two mouse models of latent TB, H56 provided significant protection against reactivation of the disease. Detailed examination showed that $\mathrm{H} 56$ promoted the generation in the lungs of antigenspecific, polyfunctional CD4 ${ }^{+} \mathrm{T}$ cells expressing interferon- $\gamma$, interleukin- 2 and tumour necrosis factor, which are thought to be important in the quality and endurance of the immune response.

Together, these data show that the inclusion of an antigen that is also expressed in the late stages of TB can enhance containment and prevent reactivation of the disease. The Statens Serum Institut in Copenhagen, Denmark, has reported that $\mathrm{H} 56$ will enter clinical trials in South Africa in March 2011.

\section{Man Tsuey Tse}

ORIGINAL RESEARCH PAPER Aagaard, C. et al. A multistage tuberculosis vaccine that confers efficient protection before and after exposure. Nature Med. 17, 189-194 (2011) 